Research Journal of Applied Sciences, Engineering and Technology 6(10): 1749-1756, 2013

DOI:10.19026/rjaset.6.3898

ISSN: 2040-7459; e-ISSN: 2040-7467

(C) 2013 Maxwell Scientific Publication Corp.

Submitted: October 17, $2012 \quad$ Accepted: December 10, 2012

Published: July 20, 2013

\title{
Research Article \\ Homogeneous Turbulence in a First-order Reactant for the Case of Multi-point and Multi-time Prior to the Final Period of Decay in a Rotating System
}

\author{
M.A. Bkar PK, M.S. Alam Sarker and M.A.K. Azad \\ Department of Applied Mathematics, University of Rajshahi-6205, Bangladesh
}

\begin{abstract}
Using Deissler's approach, the decay for the concentration of a dilute contaminant undergoing a firstorder chemical reaction in homogeneous turbulence at times prior to the final period of decay in a rotating system for the case of multi-point and multi-time is studied. Here two and three point correlations between fluctuating quantities have been considered and the quadruple correlations are ignored in comparison to the second and third order correlations. Taking Fourier transform the correlation equations are converted to spectral form. Finally, integrating the energy spectrum over all wave numbers we obtained the decay law for the concentration fluctuations in a homogeneous turbulence prior to the final period in a rotating system for the case of multi-point and multi-time.
\end{abstract}

Keywords: Deissler's method, dust particle, first order reactant, Navier-stock's equation, turbulent flow

\section{INTRODUCTION}

The mathematical models that describe chemical reaction kinetics provide chemists and chemical engineers with tools to better understand and describe chemicals processes such as food decomposition, stratospheric ozone decomposition and the complex chemistry of biological systems. The essential characteristic of turbulent flows is that turbulent fluctuations are random in nature. In geophysical flows, the system is usually rotation with a constant velocity; such large scale flows are generally turbulent. The Coriolis Effect is caused by the rotation of the Earth and the inertia of the mass experiencing the effect. The most commonly encountered rotating reference frame in the Earth. Because the Earth completes only one rotation per day, this force causes moving objects on the surface of the Earth to appear to change direction to the right in the northern hemisphere and to the left in the southern and it has great significance in Astrophysics, Stellar dynamics, Earth sciences.

Meteorology, physical geology and oceanography: Following Deissler's approaches (Deissler, 1958, 1960) the two-point; two-time correlations are obtained by considering the equation for the concentration of a dilute contaminant undergoing a first order chemical reaction. In order to solve the equations for the final period, the triple order correlation terms are neglected in comparison to the second-order ones. Loeffler and Deissler (1961) used the theory, developed by Deissler $(1958,1960)$ to study the temperature fluctuations in homogeneous turbulence before the final period. In the study of homogeneous fluid turbulence a method is describing theoretically the concentration fluctuations of dilute contaminant a first order reactant prior to the ultimate phage of decay by Kumar and Patel (1974) they studied the first order reactants in homogeneous turbulence for the case of multi-point and single time consideration. Kumar and Patel (1975) extended their problem (1974) for the case of multi-point and multitime concentration correlation. In Sarker and Kishore (1991), studied the decay of MHD turbulence at times before the final period using Chandrasekhar's relation (Chandrasekhar, 1951). Islam and Sarker (2001) studied the first-order reactant in MHD turbulence before the final period of decay for the case of multi-point and multi-time. PK et al. (2012) studied the first-order reactant in homogeneous dusty fluid turbulence prior to the ultimate phase of decay for four-point correlation in a rotating system. Corrsin (1951) obtained the spectrum of isotropic temperature fluctuations in isotropic turbulence.

In our present study, we studied the fluctuation of concentration of a dilute contaminant undergoing a first-order chemical reaction in homogeneous turbulence prior to the final period of decay in a rotating system for the case of multi-point and multitime. Here, we have considered two-point and threepoint correlation equations and solved these equations after neglecting fourth-order correlation terms. Finally we obtained the decay law of energy fluctuations of concentration of dilute contaminant undergoing a first order chemical reaction in homogeneous turbulence in a rotating system for the case of multi-point and multitime comes out to the form:

$$
\left\langle X^{2}\right\rangle=\exp \left(-2 \mathrm{RT}_{\mathrm{m}}\right)\left\{A T_{m}^{-3 / 2}+\exp \left[-2 \varepsilon_{m k i} \Omega_{m}\right] B T_{m}^{-5}\right\}
$$


where, $\left\langle X^{2}\right\rangle$ denotes the concentration fluctuation energy. It is seen that the demolition of the impurity is more rapid than that in the case of pure mixing. This result has been shown in the figure also.

\section{MATERIALS AND METHODS}

Basic equation: The differential equation governing the concentration of a dilute contaminant undergoing a first-order chemical reaction in homogeneous fluid turbulence in a rotating system could be written as:

$$
\frac{\partial u_{i}}{\partial t}+u_{k} \frac{\partial u_{i}}{\partial x_{k}}=-\frac{1}{\rho} \frac{\partial p}{\partial x_{k}}+v \frac{\partial^{2} u_{i}}{\partial x_{k} \partial x_{k}}-R u_{i}-2 \varepsilon_{m k i} \Omega_{m} u_{i i}
$$

The subscripts can take on the values 1, 2 and 3 .

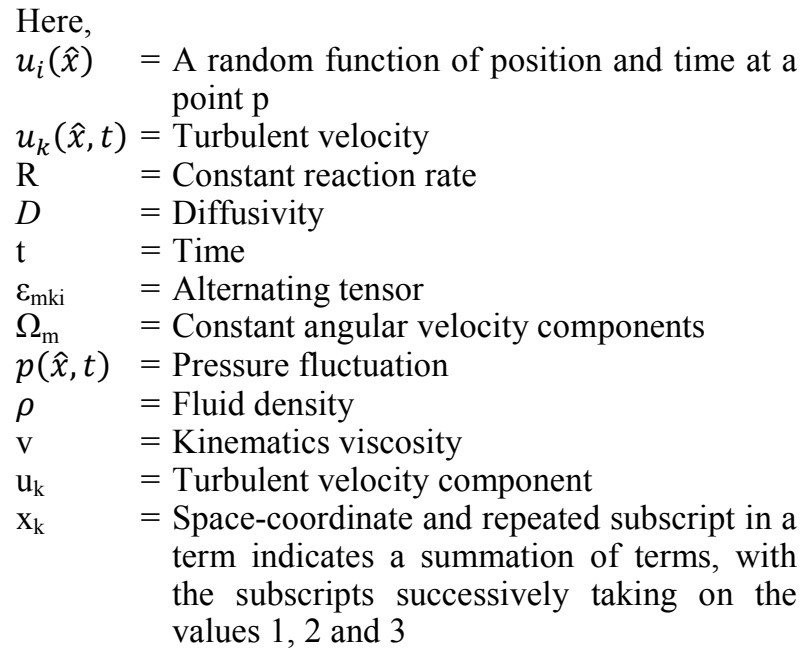

Tow-point, two-time correlation and spectral equations: Under the restrictions that:

- The turbulence and the concentration fields are homogeneous

- The chemical reaction and the local mass transfer have no effect on the velocity field

- The reaction rate and the diffusivity are constant, differential equation governing the concentration of a dilute contaminant undergoing a first-order chemical reaction we take the Navier-Stokes equations at the point $\mathrm{P}$ and the concentration equation at $\mathrm{P}^{\prime}$ and separated by the vector $\hat{r}$ could be written as:

$$
\begin{aligned}
& \frac{\partial X}{\partial t}+u_{k} \frac{\partial X}{\partial x_{k}}=D \frac{\partial^{2} X}{\partial x_{k} \partial x_{k}}-R X \\
& \frac{\partial X^{\prime}}{\partial t^{\prime}}+u_{k}^{\prime} \frac{\partial X^{\prime}}{\partial x_{k}^{\prime}}=D \frac{\partial^{2} X^{\prime}}{\partial x_{k}^{\prime} \partial x_{k}^{\prime}}-R X^{\prime}
\end{aligned}
$$

where, $X(\hat{x}, t)$ is a random function of position and time. The other symbols are as usual.
Multiplying Eq. (2) by $X^{\prime}$, Eq. (3) by $X$ and averaging, we get:

$$
\begin{aligned}
& \frac{\partial\left\langle X X^{\prime}\right\rangle}{\partial t}+\frac{\partial\left\langle u_{k} X X^{\prime}\right\rangle}{\partial x_{k}}=D \frac{\partial^{2}\left\langle X X^{\prime}\right\rangle}{\partial x_{k} \partial x_{k}}-R\left\langle X X^{\prime}\right\rangle \\
& \frac{\partial\left\langle X X^{\prime}\right\rangle}{\partial t^{\prime}}+\frac{\partial\left\langle u_{k}^{\prime} X X^{\prime}\right\rangle}{\partial x_{k}^{\prime}}=D \frac{\partial^{2}\left\langle X X^{\prime}\right\rangle}{\partial x_{k}^{\prime} \partial x_{k}^{\prime}}-R\left\langle X X^{\prime}\right\rangle
\end{aligned}
$$

where, the conditions of continuity and the fact that the quantities at a point at a particular time are independent of the positions at the other points have been utilized. Using the transformations:

$$
\frac{\partial}{\partial x_{k}}=-\frac{\partial}{\partial r_{k}}, \frac{\partial}{\partial x_{k}^{\prime}}=\frac{\partial}{\partial r_{k}}, \frac{\partial}{\partial t}=\left(\frac{\partial}{\partial t}\right)_{\Delta t}-\frac{\partial}{\partial \Delta t}, \frac{\partial}{\partial t^{\prime}}=\frac{\partial}{\partial \Delta t}
$$

in to Eq. (4) and (5), we obtains:

$$
\begin{aligned}
& \frac{\partial\left\langle X X^{\prime}\right\rangle}{\partial t}+\frac{\partial\left\langle u_{k} X X^{\prime}\right\rangle}{\partial r_{k}}(-\hat{r},-\Delta t, t+\Delta t)- \\
& \frac{\partial\left\langle u_{k} X X^{\prime}\right\rangle}{\partial r_{k}}(\hat{r}, \Delta t, t)=2 D \frac{\partial^{2}\left\langle X X^{\prime}\right\rangle}{\partial r_{k} \partial r_{k}}-2 R\left\langle X X^{\prime}\right\rangle \\
& \frac{\partial\left\langle X X^{\prime}\right\rangle}{\partial \Delta t}+\frac{\partial\left\langle u_{k} X X^{\prime}\right\rangle}{\partial r_{k}}(-\hat{r},-\Delta t, t+\Delta t)=D \frac{\partial^{2}\left\langle X X^{\prime}\right\rangle}{\partial r_{k} \partial r_{k}}-R\left\langle X X^{\prime}\right\rangle
\end{aligned}
$$

In order to reduce Eq. (6) and (7) to spectral form by using three-dimensional Fourier transform:

$$
\begin{aligned}
& \left\langle X X^{\prime}(\hat{r}, \Delta t, t)\right\rangle=\int_{-\infty}^{\infty} \theta(\hat{k}, \Delta t, t) \exp (i \hat{k} . \hat{r}) d \hat{k} \\
& \left\langle X X^{\prime}(\hat{r}, \Delta t, t)\right\rangle=\int_{-\infty}^{\infty} \phi_{k}(\hat{k}, \Delta t, t) \exp (i \hat{k} . \hat{r}) d \hat{k}
\end{aligned}
$$

We get:

$$
\begin{aligned}
& \frac{\partial \theta}{\partial t}+\left(2 D k^{2}+2 R\right) \theta=i k_{k} \phi_{k}(\hat{k}, \Delta t, t) \\
& +i\left(-k_{k}\right) \phi_{k}\left(-\hat{k}_{k},-\Delta t, t+\Delta t\right) \\
& \frac{\partial \theta}{\partial \Delta t}+\left(D k^{2}+R\right) \theta=-i k_{k} \phi_{k}(-\hat{k},-\Delta t, t+\Delta t)
\end{aligned}
$$

Solution for the ultimate phase of decomposing turbulence: For the ultimate phase of homogeneous turbulence decompose, the third-order correlations can be ignored in comparison to the second-order correlations, with this approximation, the solutions of Eq. (10) and (11) may be obtained as:

$$
\theta=f_{1}(\hat{k}, \Delta t) \exp \left[-\left(2 D k^{2}+2 R\right)\left(t-t_{0}\right)\right]
$$


Res. J. Appl. Sci. Eng. Technol., 6(10): 1749-1756, 2013

$$
\left.\theta=f_{2}(\hat{k}, t) \exp \left[-\left(D k^{2}+R\right) \Delta t\right)\right]
$$

For consistent solution of Eq. (12) and (13) we must have:

$$
G(k)=f(k) \exp \left[\left(-2 D k^{2}+2 R\right)\left(t-t_{0}+\frac{\Delta t}{2}\right)\right]
$$

where, $\mathrm{G}(\mathrm{k})=2 \pi k^{2} \theta$ is the concentration spectrum function. We evaluate $f(\mathrm{k})$ by Corrsin (1951) i.e., $f(\mathrm{k})$ $=\mathrm{N}_{\mathrm{o}} \mathrm{k}^{2} \mid \pi$. where, $\mathrm{N}_{0}=\mathrm{A}$ constant depend on initial condition. Thus, we obtain:

$$
G(k)=\frac{N_{0} k^{2}}{\pi} \exp \left[\left(-2 D k^{2}+2 R\right)\left(t-t_{0}+\frac{\Delta t}{2}\right)\right]
$$

By integrating Eq. (15) with respect to k, we obtain:

$$
\begin{aligned}
& \left\langle X X^{\prime}\right\rangle\left(\hat{r}, t_{m}\right)=\frac{N_{o} D^{1 / 2}}{4 \sqrt{4 \pi}\left(t_{m}-t_{o}\right)^{3 / 2}} \exp \\
& \left\{-\left[\frac{2 C\left(t_{m}-t_{o}\right)+r^{2}}{8 D\left(t_{m}-t_{o}\right)}\right]\right\}
\end{aligned}
$$

where, $\mathrm{t}_{\mathrm{m}}=\mathrm{t}+\Delta \mathrm{t} / 2$.

Three-point, three-time correlation and equations: Under the same assumptions as before, we take the Navier-Stokes equation for homogeneous fluid turbulence in a rotating system at the point $P$ and the concentration equations at $\mathrm{P}^{\prime}$ and $\mathrm{P}^{\prime \prime}$ as:

$$
\begin{aligned}
& \frac{\partial u_{i}}{\partial t}+u_{k} \frac{\partial u_{i}}{\partial x_{k}}=-\frac{1}{\rho} \frac{\partial p}{\partial x_{k}}+v \frac{\partial^{2} u_{i}}{\partial x_{k} \partial x_{k}}-R u_{i}-2 \varepsilon_{m k i} \Omega_{m} u_{i}(17 \\
& \frac{\partial X^{\prime}}{\partial t^{\prime}}+u_{k}^{\prime} \frac{\partial X^{\prime}}{\partial x_{k}^{\prime}}=D \frac{\partial^{2} X^{\prime}}{\partial x_{k}^{\prime} \partial x_{k}^{\prime}}-R X^{\prime} \\
& \frac{\partial X^{\prime \prime}}{\partial t^{\prime \prime}}+u_{k}^{\prime \prime} \frac{\partial X^{\prime \prime}}{\partial x_{k}^{\prime \prime}}=D \frac{\partial^{2} X^{\prime \prime}}{\partial x_{k}^{\prime \prime} \partial x_{k}^{\prime \prime}}-R X^{\prime \prime}
\end{aligned}
$$

Multiplying Eq. (17) by $\mathrm{X}^{\prime} \mathrm{X}^{\prime \prime}$, (18) by $\mathrm{u}_{\mathrm{i}} \mathrm{X}^{\prime \prime}$ and (19) by $u_{i} X^{\prime}$ and then taking space averages, we obtain:

$$
\begin{aligned}
& \frac{\partial}{\partial t}\left\langle u_{i} X^{\prime} X^{\prime \prime}\right\rangle^{+} \\
& \frac{\partial}{\partial x_{k}}\left\langle u_{i} u_{k} X^{\prime} X^{\prime \prime}\right\rangle=-\frac{1}{\rho} \frac{\partial}{\partial x_{k}}\left\langle p X^{\prime} X^{\prime \prime}\right\rangle+v \frac{\partial^{2}}{\partial x_{k} \partial x_{k}}\left\langle u_{i} X^{\prime} X^{\prime \prime}\right\rangle \\
& -R\left\langle u_{i} X^{\prime} X^{\prime \prime}\right\rangle-2 \varepsilon_{m k i} \Omega_{m}\left\langle u_{i} X^{\prime} X^{\prime \prime}\right\rangle \\
& \frac{\partial}{\partial t^{\prime}}\left\langle u_{i} X^{\prime} X^{\prime \prime}\right\rangle+
\end{aligned}
$$

$$
\frac{\partial}{\partial x_{k}^{\prime}}\left\langle u_{i} u_{k}^{\prime} X^{\prime} X^{\prime \prime}\right\rangle=D \frac{\partial^{2}}{\partial x_{k}^{\prime} \partial x_{k}^{\prime}}\left\langle u_{i} X^{\prime} X^{\prime \prime}\right\rangle-R\left\langle\mathrm{u}_{\mathrm{i}} \mathrm{X}^{\prime} \mathrm{X}^{\prime \prime}\right\rangle
$$

$$
\begin{aligned}
& \frac{\partial}{\partial t^{\prime \prime}}\left\langle u_{i} X^{\prime} X^{\prime \prime}\right\rangle+ \\
& \frac{\partial}{\partial x_{k}^{\prime \prime}}\left\langle u_{i} u_{k}^{\prime \prime} X^{\prime} X^{\prime \prime}\right\rangle=D \frac{\partial^{2}}{\partial x_{k}^{\prime \prime} \partial x_{k}^{\prime \prime}}\left\langle u_{i} X^{\prime} X^{\prime \prime}\right\rangle-R\left\langle\mathrm{u}_{\mathrm{i}} \mathrm{X}^{\prime} \mathrm{X}^{\prime \prime}\right\rangle
\end{aligned}
$$

Using the transformations:

$$
\begin{aligned}
& \frac{\partial}{\partial x_{k}}=-\left(\frac{\partial}{\partial r_{k}}+\frac{\partial}{\partial r_{k}^{\prime}}\right), \frac{\partial}{\partial x_{k}^{\prime}}=\frac{\partial}{\partial r_{k}}, \frac{\partial}{\partial x_{k}^{\prime \prime}}=\frac{\partial}{\partial r_{k}^{\prime}}, \frac{\partial}{\partial t} t^{\prime}, t^{\prime \prime} \\
& =\left(\frac{\partial}{\partial t}\right)_{\Delta t, \Delta t^{\prime}}-\frac{\partial}{\partial \Delta t}-\frac{\partial}{\partial \Delta t^{\prime}}, \frac{\partial}{\partial t^{\prime}}=\frac{\partial}{\partial \Delta t}, \frac{\partial}{\partial t^{\prime \prime}}=\frac{\partial}{\partial \Delta t^{\prime}}
\end{aligned}
$$

Into Eq. (20) to (22), we get:

$$
\begin{aligned}
& \frac{\partial}{\partial t}\left\langle u_{i} X^{\prime} X^{\prime \prime}\right\rangle-\left(\frac{\partial}{\partial r_{k}}+\frac{\partial}{\partial r_{k}^{\prime}}\right)\left\langle u_{i} u_{k} X^{\prime} X^{\prime \prime}\right\rangle \\
& +\frac{\partial}{\partial r_{k}}\left\langle u_{i} u_{k}^{\prime} X^{\prime} X^{\prime \prime}\right\rangle+\frac{\partial}{\partial r_{k}^{\prime}}\left\langle u_{i} u_{k}^{\prime \prime} X^{\prime} X^{\prime \prime}\right\rangle \\
& =-\frac{1}{\rho}\left(\frac{\partial}{\partial r_{k}}+\frac{\partial}{\partial r_{k}^{\prime}}\right)\left\langle p X^{\prime} X^{\prime \prime}\right\rangle+v\left(\frac{\partial}{\partial r_{k}}+\frac{\partial}{\partial r_{k}^{\prime}}\right)^{2} \\
& \left\langle u_{i} X^{\prime} X^{\prime \prime}\right\rangle+D\left(\frac{\partial^{2}}{\partial r_{k} \partial r_{k}}+\frac{\partial^{2}}{\partial r_{k}^{\prime} \partial r_{k}^{\prime}}\right)\left\langle u_{i} X^{\prime} X^{\prime \prime}\right\rangle \\
& -\left(2 R+2 \varepsilon_{m k i} \Omega_{m}\right)\left\langle u_{i} X^{\prime} X^{\prime \prime}\right\rangle \\
& \frac{\partial}{\partial \Delta t}\left\langle u_{i} X^{\prime} X^{\prime \prime}\right\rangle+\frac{\partial}{\partial r_{k}}\left\langle u_{i} u_{k}^{\prime} X^{\prime} X^{\prime \prime}\right\rangle \\
& =D \frac{\partial^{2}}{\partial r_{k}^{\prime} \partial r_{k}^{\prime}}\left\langle u_{i} X^{\prime} X^{\prime \prime}\right\rangle-R\left\langle X^{\prime} u_{i} X^{\prime \prime}\right\rangle \\
& \frac{\partial}{\partial \Delta t^{\prime}}\left\langle u_{i} X^{\prime} X^{\prime \prime}\right\rangle+\frac{\partial}{\partial r_{k}^{\prime}}\left\langle u_{i} u_{k}^{\prime \prime} X^{\prime} X^{\prime \prime}\right\rangle \\
& =D \frac{\partial^{2}}{\partial r_{k}^{\prime} \partial r_{k}^{\prime \prime}}\left\langle u_{i} X^{\prime} X^{\prime \prime}\right\rangle-R\left\langle X^{\prime} u_{i} X^{\prime \prime}\right\rangle
\end{aligned}
$$

Using the six-dimensional Fourier transform of the type:

$$
\begin{aligned}
& \left\langle X X^{\prime} u_{k}^{\prime \prime}\left(\hat{r}, \hat{r}^{\prime}, \Delta t, \Delta t^{\prime}, t\right)\right\rangle \\
& =\int_{-\infty}^{\infty} \cdot \int_{-\infty}^{\infty} \psi_{k}\left(\hat{k}, \hat{k}^{\prime}, \Delta t, \Delta t^{\prime}, t\right) \exp \left(\hat{k} \cdot \hat{r}+\hat{k}^{\prime} \cdot \hat{r}^{\prime}\right) d \hat{k} d \hat{k}^{\prime}
\end{aligned}
$$

and the assumption that the quintuple correlations representing the transfer terms in Eq. (23) to (25) can be neglected as they decay faster than the lower-order 
Res. J. Appl. Sci. Eng. Technol., 6(10): 1749-1756, 2013

correlation terms. Then the Eq. (23) to (25) in Fourier space can be written as:

$$
\begin{aligned}
& \frac{\partial \psi_{i}}{\partial t}\left(\hat{k}, \hat{k}^{\prime}, \Delta t, \Delta t^{\prime}, t\right)+D\left[\left(1+N_{S}\right) k^{2}+2 N_{s} k k^{\prime} \cos \left\{\left(1+N_{s}\right) k^{\prime 2}\right.\right. \\
& \left.\left.+\left(2 R+2 \varepsilon_{m k i} \Omega_{m}\right) / D\right\}\right] \psi_{i}\left(\hat{k}, \hat{k}^{\prime}, \Delta t, \Delta t^{\prime}, t\right)=0 \\
& \frac{\partial \psi_{i}}{\partial \Delta t}\left(\hat{k}, \hat{k}^{\prime}, \Delta t, \Delta t^{\prime}, t\right)+D[ \\
& \left.\left(k^{2}+2 R\right) / D\right] \psi_{i}\left(\hat{k}, \hat{k}^{\prime}, \Delta t, \Delta t^{\prime}, t\right)=0 \\
& \frac{\partial \psi_{i}}{\partial \Delta t^{\prime}}\left(\hat{k}, \hat{k}^{\prime}, \Delta t, \Delta t^{\prime}, t\right)+D[ \\
& \left.\left(k^{\prime 2}+2 R\right) / D\right] \psi_{i}\left(\hat{k}, \hat{k}^{\prime}, \Delta t, \Delta t^{\prime}, t\right)=0
\end{aligned}
$$

where, $N_{s}=v / D$, the Schmidt number

As the pressure force terms are related to higherorder correlations, therefore, these along with the quadruple correlations are also neglected.

Integrating Eq. (26) to (28) between $t_{o}$ and $t$, we obtain:

$$
\begin{aligned}
& \psi_{i}=f_{i} \exp \left\{-D\left[\left(1+N_{s}\right) k^{2}+2 N_{s} k k^{\prime}\right.\right. \\
& \left.\left.\cos \theta+\left(1+N_{s}\right) k^{\prime 2}+\left(2 R+2 \varepsilon_{m k i} \Omega_{m}\right) / D\right]\left(t-t_{0}\right)\right\} \\
& \psi_{i}=g_{i} \exp \left\{\left[-D\left(k^{2}+R / D\right)\right] \Delta t\right\} \\
& \psi_{i}=h_{i} \exp \left\{\left[-D\left(k^{\prime 2}+R / D\right)\right] \Delta t^{\prime}\right\}
\end{aligned}
$$

For these relations to be consistent, we have:

$$
\begin{gathered}
k_{i} \psi_{i}=k_{i}\left(\psi_{i}\right)_{0} \exp \left[-D\left\{\left(1+N_{s}\right)\left(k^{2}+k^{\prime 2}\right)\right.\right. \\
\left(t-t_{0}\right)+k^{2} \Delta t+k^{\prime 2} \Delta t^{\prime}+2 N_{s} k k^{\prime} \cos \theta\left(t-t_{0}\right) \\
\left.\left.+(2 R / D)\left[t-t_{0}+\left(\Delta t+\Delta t^{\prime}\right) / 2\right]+\left[\left(2 \varepsilon_{m k i} \Omega_{m}\right) / D\right]\left(t-t_{0}\right)\right\}\right]
\end{gathered}
$$

where, the subscript 0 refers to the value of $\psi_{\mathrm{i}}$ at $t=t_{o}$, $\Delta \mathrm{t}=\Delta \mathrm{t}^{\prime}=0$ and $\theta$ is the angle between $k$ and $\mathrm{k}^{\prime}$. The relation between $\phi_{\mathrm{i}}$ and $\psi_{\mathrm{i}}$ is given by:

$$
k_{i} \phi_{i}(\hat{k}, \Delta t, t)=\int_{-\infty}^{\infty} k_{i} \psi_{i}\left(\hat{k}, \Delta t, \hat{k}^{\prime}, 0, t\right) d k^{\prime}
$$

Substituting Eq. (30) and (29) into Eq. (10), we obtain:

$$
\frac{\partial G}{\partial t}+\left(2 k^{2} D+2 R\right) G=W
$$

where, $G=2 \pi \mathrm{k}^{2} \theta$ and

$$
\begin{aligned}
& \left.W=\int_{0}^{\infty} i k_{i}\left(\psi_{i}\right)\right)_{0} k^{2} k^{\prime 2}(2 \pi)^{2} \exp \\
& \left\{-\mathrm{D}\left[\left(1+\mathrm{N}_{\mathrm{S}}\right) \mathrm{k}^{2}\left(\mathrm{t}-\mathrm{t}_{\mathrm{o}}+\frac{\Delta \mathrm{t}}{\mathrm{t}+\mathrm{N}_{\mathrm{S}}}\right)+\mathrm{k}^{\prime 2}\left(1+\mathrm{N}_{\mathrm{S}}\right)\left(\mathrm{t}-\mathrm{t}_{\mathrm{o}}\right)\right.\right. \\
& +\left[\left(2 \varepsilon_{m k i} \Omega_{m}\right) / D\right]\left(t-t_{0}\right) \\
& +(2 R / D) \\
& \left.\left.\left(t-t_{o}+\frac{\Delta t}{2}\right)\right\}\right] \int_{-1}^{1} \exp \left[-2 N_{s} D k k^{\prime}\left(t-t_{0}\right) \cos \theta\right] d k^{\prime} \\
& +\int_{0}^{\infty}\left[i\left(-k_{i}\right) \psi_{i}\left(-\hat{k}_{,}-\hat{k}^{\prime}\right)\right]_{0}(2 \pi)^{2} k^{2} k^{\prime 2} \exp \{-D \\
& {\left[\left(1+N_{S}\right) k^{2}\left(t-t_{o}+\frac{\Delta t}{1+N_{S}}\right)\right.} \\
& -k^{\prime 2}\left(1+N_{s}\right)\left(t-t_{0}+\Delta t\right)+\left[\left(2 \varepsilon_{m k i} \Omega_{m}\right) / D\right]\left(t-t_{0}\right) \\
& \left.\left.+\frac{2 R}{D}\left(t-t_{0}+\frac{\Delta t}{2}\right)\right]\right\} \\
& \times \int_{-1}^{1} \exp \left[-2 N_{s} D k k^{\prime}\left(t-t_{0}+\Delta t\right) \cos \theta\right](d \cos \theta) d k^{\prime}(32)
\end{aligned}
$$

where, $\mathrm{dk}^{\prime}$ is written as $2 \pi k^{\prime 2} d(\cos \theta) d k^{\prime}$ and the quantity $\left(\psi_{\mathrm{i}}\right)_{0}$ depends on the initial conditions of the turbulence. Now, following Deissler (1958, 1960), we take:

$$
(2 \pi)^{2} i\left[k_{i} \psi_{i}\left(\hat{k}, \hat{k}^{\prime}\right)_{0}=-\frac{1}{2} \delta_{0}\left(k^{2} k^{\prime 4}-k^{4} k^{\prime 2}\right)\right.
$$

and

$$
(2 \pi)^{2} i\left[-k_{i} \psi_{i}\left(-\hat{k},-\hat{k}^{\prime}\right)_{0}=\frac{1}{2} \delta_{0}\left(k^{2} k^{\prime 4}-k^{4} k^{\prime 2}\right)\right.
$$

Substituting Eq. (33) in (32) and completing the integration, we get:

$$
\begin{aligned}
& W=-\frac{\delta_{o} N_{s} \pi^{1 / 2}}{4 D^{3 / 2}\left(t-t_{o}\right)^{3 / 2}\left(1+N_{s}\right)^{5 / 2}} \\
& \exp \left[-k^{2} D \frac{1+2 N_{s}}{1+N_{s}}\left(t-t_{o}+\frac{1+N_{s}}{1+2 N_{s}} \Delta t\right)\right.
\end{aligned}
$$




$$
\begin{aligned}
& \left.+\left(2 \varepsilon_{m k i} \Omega_{m}\right)\left(t-t_{0}\right)-2 R\left(t-t_{o}+\frac{\Delta t}{2}\right)\right] \\
& \left\{\frac{15 k^{4}}{4 N_{s}^{2}\left(t-t_{o}\right)^{2} D^{2}} \frac{N_{s}}{1+N_{s}}+\left[5\left(\frac{N_{s}}{1+N_{s}}\right)^{2}-\frac{3}{2}\right]\right. \\
& \left.\times \frac{k^{6}}{N_{s} D\left(t-t_{o}\right)}+\left[\left(\frac{N_{s}}{1+N_{s}}\right)^{3}-\frac{N_{s}}{1+N_{s}}\right] k^{8}\right\} \\
& -\frac{\delta o N_{s} \sqrt{\pi}}{4 D^{3} / 2\left(t-t_{o}+\Delta t\right)^{3 / 2}\left(t+N_{S}\right)^{5 / 2}} \\
& \times \exp \left[\begin{array}{l}
-k^{2} D \frac{1+2 N_{s}}{1+N_{s}}\left(t-t_{o}+\frac{N_{s}}{1+N_{s}} \Delta t\right) \\
+\left(2 \varepsilon_{m k i} \Omega_{m}\right)\left(t-t_{0}\right)-2 R\left(t-t_{o}-\frac{\Delta t}{2}\right)
\end{array}\right] \\
& \times\left\{\frac{15 k^{4}}{4 D^{2} N_{s}^{2}\left(t-t_{o}+\Delta t\right)^{2}}\left(\frac{N_{s}}{1+N_{s}}\right)+\left[\begin{array}{l}
5\left(\frac{N_{s}}{1+N_{s}}\right) \\
2-\frac{3}{2}
\end{array}\right] \frac{k^{6}}{N_{s} D\left(t-t_{o}+\Delta t\right)}\right. \\
& \left.+\left[\left(\frac{N_{s}}{1+N_{s}}\right)^{3}-\frac{N_{s}}{1+N_{s}}\right] k^{8}\right\}
\end{aligned}
$$

This represents the transfer function arising due to the consideration of concentration at three-point and three-time. When $\Delta \mathrm{t}=0$ and $R=0$, the expression for reduces to the case of pure mixing. It may also be noted that (for $\Delta \mathrm{t}=0$ ):

$$
\int_{0}^{\infty} W d k=0
$$

This means that the conditions of continuity and homogeneity are satisfied. Physically, it was to be expected as $W$ is a measure of the energy transfer and the total energy transferred to all wave numbers must be zero.

With the help of Eq. (31) and (34), one can get:

$$
\begin{aligned}
\mathrm{G} & =\frac{N_{0} k^{2}}{\pi} \exp \left\{-2\left(k^{2} D+2 R\right)\left(t-t_{o}+\frac{\Delta t}{2}\right)\right\}+\frac{\sqrt{\pi} N_{s}}{D^{3 / 2}\left(1+N_{s}\right)^{7 / 2}} \\
& \frac{\delta_{0}}{4} \times \exp \left[\begin{array}{l}
-D \frac{1+2 N_{s}}{1+N_{s}} k^{2}\left(t-t_{o}+\frac{\left(1+N_{s}\right) \Delta t}{1+2 N_{s}}\right) \\
+\left(2 \varepsilon_{m k i} \Omega_{m}\right)\left(t-t_{0}\right)-2 R\left(t-t_{o}+\frac{\Delta t}{2}\right)
\end{array}\right] \\
& \times\left[\begin{array}{l}
\frac{3 k^{4}}{2 N_{s} D^{2}\left(t-t_{o}\right)^{5 / 2}}+\frac{\left(7 N_{s}-6\right) k^{6}}{3 D\left(1+N_{s}\right)\left(t-t_{o}\right)^{3 / 2}} \\
-\frac{4\left(3 N_{s}^{2}-2 N_{s}+3\right) k^{8}}{3\left(1+N_{s}\right)^{2}\left(t-t_{o}\right)^{1 / 2}}
\end{array}\right.
\end{aligned}
$$

$$
\begin{aligned}
& \left.+\frac{8 D^{1 / 2}\left(3 N_{s}^{2}-2 N_{s}+3\right) k^{9}}{3\left(1+N_{s}\right)^{5 / 2}} F\left(k \sqrt{\frac{\left(t-t_{o}\right) D}{1+N_{s}}}\right)\right] \\
& +\frac{\sqrt{\pi} N_{s}}{D^{3 / 2}\left(1+N_{s}\right)^{7 / 2}} \frac{\delta_{o}}{4} \\
& \times \exp \left[-D\left(\frac{1+2 N_{s}}{1+N_{s}}\right) k^{2}\left(t-t_{o}+\frac{N_{s}}{1+2 N_{s}} \Delta t\right)\right] \\
& \times\left[\frac{\left.\left.3 \varepsilon_{m k i} \Omega_{m}\right)\left(t-t_{0}\right)-2 R\left(t-t_{o}+\frac{\Delta t}{2}\right)\right]}{2 D^{2} N_{s}\left(t-t_{o}+\Delta t\right)^{5 / 2}}+\frac{\left(7 N_{s}-6\right) k^{6}}{3 D\left(1+N_{s}\right)\left(t-t_{o}+\Delta t\right)^{3 / 2}}\right. \\
& -\frac{4}{3} \frac{\left(3 N_{s}^{2}-2 N_{s}+3\right) k^{8}}{\left(1+N_{s}\right)^{2}\left(t-t_{o}+\Delta t\right)^{1 / 2}} \\
& \left.+\frac{8 D^{1 / 2}\left(3 N_{s}^{2}-2 N_{s}+3\right) k^{9}}{\left(1+N_{s}\right)^{5 / 2}} F\left(k \sqrt{\frac{\left(t-t_{o}+\Delta t\right) D}{1+N_{s}}}\right)\right](36)
\end{aligned}
$$

where,

$$
\begin{aligned}
& F(\omega)=\exp \left(-\omega^{2}\right) \int_{0}^{\omega} \exp \left(x^{2}\right) d x \\
& \omega=\mathrm{k} \sqrt{\frac{\left(t-t_{o}\right) D}{1+N_{s}}} \text { or } \mathrm{k}=\sqrt{\frac{\left(\mathrm{t}-\mathrm{t}_{\mathrm{o}}+\Delta t\right) \mathrm{D}}{1+N_{s}}}
\end{aligned}
$$

As in the previous section, by integrating Eq. (36) with respect to $k$, we obtain:

$$
\begin{aligned}
& \left\langle\frac{X X^{\prime}}{2}\left(\Delta t, t_{m}\right)\right\rangle=\int_{0}^{\infty} G d k= \\
& \frac{N_{o}}{8 D^{3 / 2} \sqrt{2 \pi}\left(T+\frac{\Delta t}{2}\right)^{3 / 2}} \exp \left[-2 R\left(T+\frac{\Delta t}{2}\right)\right]
\end{aligned}
$$$$
+\frac{\pi}{D^{6}\left(1+N_{s}\right)\left(1+N_{s}\right)^{5 / 2}} \exp \left[-\left(2 \varepsilon_{m k l} \Omega_{m}\right)\right] \exp \left[-2 R\left(T+\frac{\Delta t}{2}\right)\right]
$$$$
\times \frac{\delta_{0}}{4}
$$$$
\frac{9}{16 T^{5 / 2}\left(T+\frac{1+N_{s}}{1+2 N_{s}} \Delta T\right)^{5 / 2}}+\frac{9}{16(T+\Delta T)^{5 / 2}\left(T+\frac{1+N_{s}}{1+2 N_{s}} \Delta T\right)^{5 / 2}}
$$$$
+\frac{5 N_{s}\left(7 N_{s}-6\right)}{16\left(1+2 N_{s}\right) T^{3 / 2}\left(T+\frac{1+N_{s}}{1+2 N_{s}} \Delta T\right)^{7 / 2}}
$$ 


$$
\begin{aligned}
& +\frac{5 N_{s}\left(7 N_{s}-6\right)}{16\left(1+2 N_{s}\right)(T+\Delta T)^{3 / 2}\left(T+\frac{N_{s}}{1+2 N_{s}} \Delta T\right)^{7 / 2}} \\
& +\frac{35 N_{s}\left(3 N_{s}^{2}-2 N_{s}+3\right)}{8\left(1+2 N_{s}\right) T^{1 / 2}\left(T+\frac{1+N_{s}}{1+2 N_{s}} \Delta T\right)^{9 / 2}} \\
& +\frac{35 N_{s}\left(3 N_{s}^{2}-2 N_{s}+3\right)}{8\left(1+2 N_{s}\right)(T+\Delta T)^{1 / 2}\left(T+\frac{N_{s}}{1+2 N_{s}} \Delta T\right)^{9 / 2}} \\
& +\frac{8 N_{s}\left(3 N_{s}^{2}-2 N_{s}+3\right)\left(1+2 N_{s}\right)^{5 / 2}}{3.2^{23 / 2}\left(1+N_{s}\right)^{11 / 2}} \\
& \sum_{\mathrm{n}=0}^{\infty} \frac{1.3 .5 \ldots(2 n+9)}{n !(2 n+1) 2^{2 n}\left(1+N_{s}\right)^{n}} \times \\
& \frac{T^{(2 n+1) / 2}}{\left(T+\frac{\Delta T}{2}\right)^{(2 n+11) / 2}}+\frac{(\mathrm{T}+\Delta \mathrm{T})^{(2 \mathrm{n}+1) / 2}}{\left(T+\frac{\Delta T}{2}\right)^{(2 n+1) / 2}}
\end{aligned}
$$

where, $\mathrm{T}=\mathrm{t}-\mathrm{t}_{\mathrm{o}}$. For $\mathrm{T}_{\mathrm{m}}=\mathrm{T}+\Delta \mathrm{T} / 2$ Eq. (37) becomes:

$$
\begin{aligned}
& \left\langle\frac{X X^{\prime}}{2}\left(t_{m}\right)\right\rangle=\exp \left(-2 R T_{m}\right) \frac{N_{0}}{8 \sqrt{2 \pi} D^{3 / 2} T_{m}^{3 / 2}} \\
& +\frac{\delta_{0} \pi}{4 D^{6}\left(1+N_{s}\right)\left(1+2 N_{s}\right)} \exp \left[-\left(2 \varepsilon_{m k i} \Omega_{m}\right)\right] \\
& \frac{9}{16} \frac{1}{\left(T_{m}-\frac{\Delta T}{2}\right)^{5 / 2}\left(T_{m}+\frac{\Delta T}{1+2 N_{s}}\right)^{5 / 2}} \\
& +\frac{9}{16\left(T_{m}+\frac{\Delta T}{2}\right)^{5 / 2}\left(T_{m}-\frac{\Delta T}{2}\right)^{5 / 2}} \\
& +\frac{5 N_{s}\left(7 N_{s}-6\right)}{16\left(1+2 N_{s}\right)} \frac{1}{\left(T_{m}+\frac{\Delta T}{2}\right)^{7 / 2}\left(T_{m}-\frac{\Delta T}{2}\right)^{3 / 2}} \\
& +\frac{5 N_{s}\left(7 N_{s}-6\right)}{16\left(1-2 N_{s}\right)} \frac{1}{\left(T_{m}+\frac{\Delta T}{2}\right)^{3 / 2}\left(T_{m}-\frac{\Delta T}{1+2 N_{S}}\right)^{7 / 2}}+\ldots
\end{aligned}
$$

If $\Delta t=0$, then Eq. (38) reduces to the form:

$$
\left\langle\frac{X^{2}}{2}\right\rangle=\exp \left(-2 R T_{m}\right)\left\{\begin{array}{l}
\frac{N_{o}}{8 \sqrt{2 \pi} D^{3 / 2}} \frac{1}{\mathrm{~T}_{\mathrm{m}}^{3 / 2}}+\frac{\delta_{o} \pi}{2 D^{6}\left(1+N_{s}\right)\left(1+2 N_{s}\right)} \\
\exp \left[-\left(2 \varepsilon_{m k i} \Omega_{m}\right)\right]\left[\frac{9}{16 T_{m}^{5}}+\frac{5}{16} \frac{N_{s}\left(7 N_{s}-6\right)}{\left(1+2 N_{s}\right) T_{m}^{5}}\right]
\end{array}\right\}
$$

Therefore,

$$
\begin{gathered}
\left\langle X^{2}\right\rangle=\exp \left(-2 \mathrm{RT}_{\mathrm{m}}\right) \\
{\left[\frac{N_{o}}{4 \sqrt{2 \pi} D^{3 / 2}} \frac{3}{T_{m}^{3 / 2}}+\exp \left[-\left(2 \varepsilon_{m k i} \Omega_{m}\right)\right] \frac{\delta_{o} \alpha}{D^{6}} \frac{1}{T_{m}^{5}}\right]}
\end{gathered}
$$

where,

$$
\begin{gathered}
\alpha=\frac{\pi}{\left(1+N_{s}\right)\left(1+2 N_{s}\right)} \\
{\left[\frac{9}{16}+\frac{5}{16} \frac{N_{s}\left(7 N_{s}-6\right)}{\left(1+2 N_{s}\right)}+\ldots\right]}
\end{gathered}
$$

Thus, the decay law for the concentration energy fluctuation of homogeneous dusty fluid in a rotating system prior to the ultimate phase may be written as:

$$
\left\langle X^{2}\right\rangle=\exp \left(-2 \mathrm{RT}_{\mathrm{m}}\right)\left\{A T_{m}^{-3 / 2}+\exp [-F] B T_{m}^{-5}\right\}
$$

where,

$$
A=\frac{3 N_{0}}{4 \sqrt{2 \pi} D^{3 / 2}}, B=\frac{\delta_{0} \alpha}{D^{6}}, F=2 \varepsilon_{m k i} \Omega_{m}
$$

\section{RESULTS AND DISCUSSION}

It is noted that $\mathrm{y} 1, \mathrm{y} 2, \mathrm{y} 3, \mathrm{y} 4, \mathrm{y} 5, \mathrm{y} 6$ and $\mathrm{y} 7$ are solution curves of Eq. (40) but in the absence of coriolis force $y 1, y 2, y 3, y 4, y 5, y 6, y 7$ are represented by Eq. (41) at the different values of chemical reaction respectively which indicated in the figures. In Eq. (40) we obtained the concentration fluctuation energy decay of homogeneous fluid turbulence in a rotating system. In the absence of corriolis force i.e. if the frame is nonrotating then Eq. (40) becomes:

$$
\left\langle X^{2}\right\rangle=\exp \left(-2 \mathrm{RT}_{\mathrm{m}}\right)\left\{A T_{m}^{-3 / 2}+B T_{m}^{-5}\right\}
$$

Which was obtained earlier by Kumar and Patel (1975). For large times, the last term of Eq. (41) becomes negligible and the decay law for the ultimate period becomes exp $\left(-2 \mathrm{RT}_{\mathrm{m}}\right)\left(\mathrm{AT}_{\mathrm{m}}^{-3 / 2}\right)$ which in the case of pure-mixing is similar to the law obtained by Corrsin (1951).

In Fig. 1 to 4 , we observe that the variation of chemical reaction $(R=3.5,1.75,0.875,0.44,0.22,0.11$ and 0 ) causes significant changes in the concentration fluctuation decay of energy of homogeneous fluid turbulence in presence of corriolis force $F=2.75,1.50$, 0.75 and 0 , respectively. It is mentioned that the energy decay of the fluid particles decreases with the increases of the chemical reaction $R$ and is maximum where the reaction rate is equal to zero. If the system is nonrotating i.e., the corriolis force is absence the energy decay of the fluid particles more rapidly than any 
Res. J. Appl. Sci. Eng. Technol., 6(10): 1749-1756, 2013

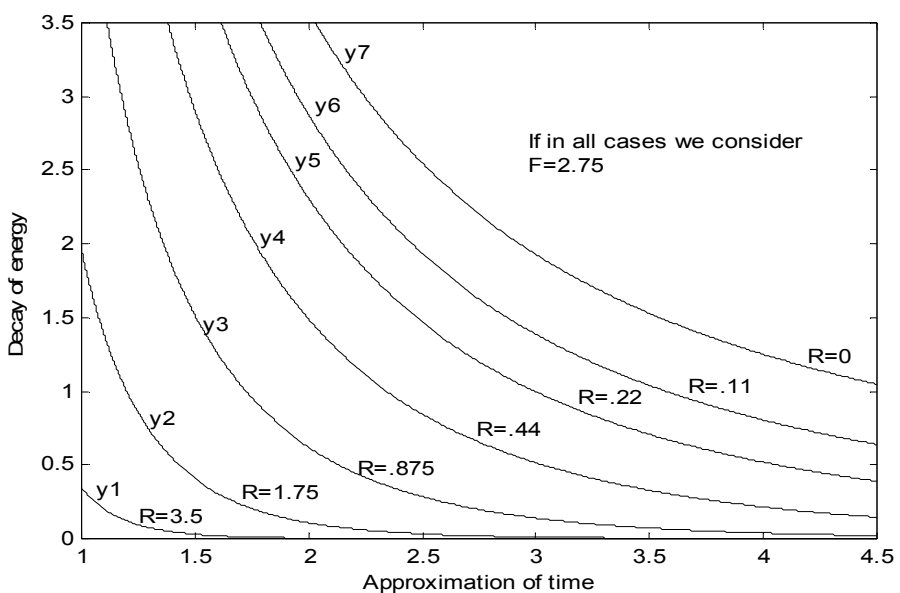

Fig. 1: Energy decay curves of Eq. (40) for $F=\exp \left(2 \varepsilon_{m k i} \Omega_{m}\right)=2.75$

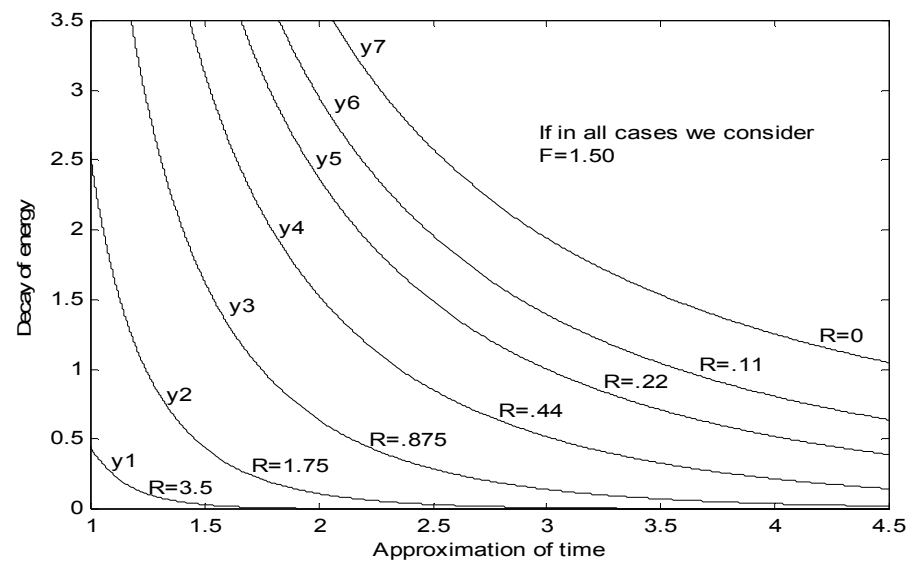

Fig. 2: Energy decay curves of Eq. (40) for $F=\exp \left(2 \varepsilon_{m k i} \Omega_{m}\right)=1.5$

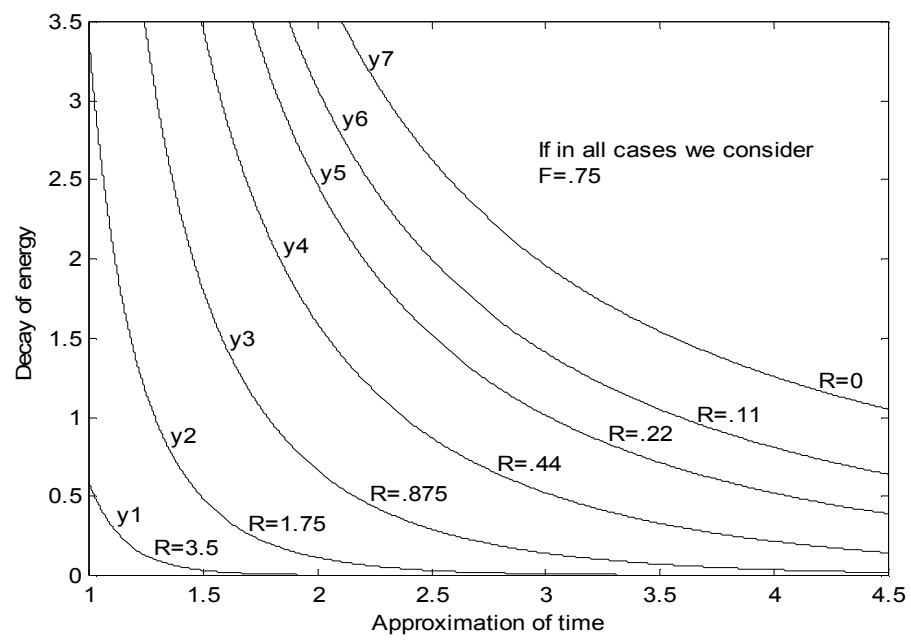

Fig. 3: Energy decay curves of Eq. (40) for $F=\exp \left(2 \varepsilon_{m k i} \Omega_{m}\right)=0.75$

rotating system which indicated in the figures below that is increasing of Coriolis force causes the energy decay of fluid particles and vice versa.
In Fig. 4, we notice that in the absence of Coriolis force the energy decay of fluid particles are maximums due to the variation of first-order chemical reaction in 


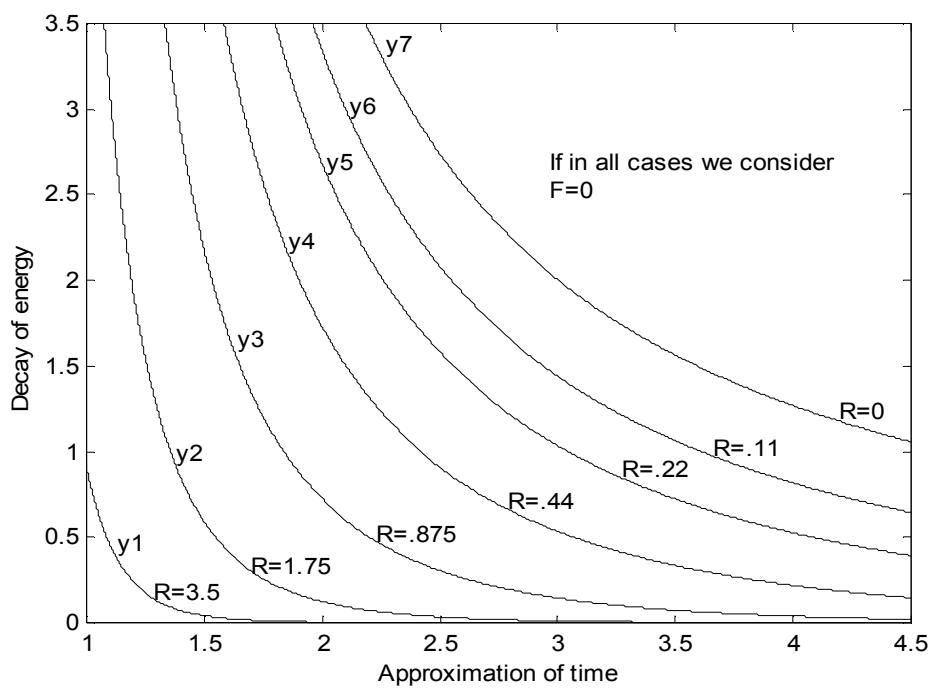

Fig. 4: Energy decay curves of Eq. (41) i.e., Eq. (40) for $F=\exp \left(2 \varepsilon_{m k i} \Omega_{m}\right)=0$

homogeneous fluid turbulence for the case of multipoint and multi-time which mention above graphically.

\section{CONCLUSION}

This study shows that if the concentration selected is the chemical reactant of the first order, then the effect is that the decomposition of the concentration fluctuation in homogeneous turbulence in the presence of Coriolis force the energy decay of the fluid particles is much more slowly if the system is non-rotating i.e., in the absence of Coriolis force. The faster rate of decomposition is governed by $\exp \left(-2 \mathrm{RT}_{\mathrm{m}}\right)$. The decomposition of the concentration fluctuation in homogeneous turbulence in the rotating system for the case of multi-point and multi-time is more slowly due to absence of Coriolis force in the first order chemical reaction. In a normal way, it takes a lot of time to get rid of a pollutant in the fluid. The only effective factor in the case of chemical reactant is exp $\left(-2 \mathrm{RT}_{\mathrm{m}}\right)$ and Coriolis force which can be taken as the correcting law for the pure mixing case and may be applied to the data for the case of reactant and thus, the numerical work required for this study has been avoided. From the above figures and discussion, we conclude that in the rotating and non-rotating frame the decomposition of the concentration fluctuation in homogeneous turbulence for the case of multi-point and multi-time are increases due to the decreases of the first order chemical reaction and maximum at the point where the chemical reaction is zero and more rapidly in the absence of coriolis force.

\section{REFERENCES}

Chandrasekhar, S., 1951. The invariant theory of isotropic turbulence in magneto-hydrodynamics. Proc. R. Soc. Lon. Ser-A, 204: 435-449.
Corrsin, S., 1951. On the spectrum of isotropic temperature fluctuations in isotropic turbulence. J. Appl. Phys., 22: 469-473.

Deissler, R.G., 1958. On the decay of homogeneous turbulence before the final period. Phys. Fluid., 1(2): 111-121.

Deissler, R.G., 1960. A theory of decaying homogeneous turbulence. Phys. Fluid., 3(2): 176-187.

Islam, M.A. and M.S.A. Sarker, 2001. First-order reactant in MHD turbulence before the final period of decay for the case of multi-point and multi-time, Indian. J. Pure Appl. Math., 32: 1173-1184.

Kumar, P. and S.R. Patel, 1974. First-order reactant in homogeneous turbulence before the final period of decay. Phys. Fluid., 17: 1362-1368.

Kumar, P. and S.R. Patel, 1975. On first-order reactants in homogeneous turbulence. Int. J. Eng. Sci., 13: 305-315.

Loeffler, A.L. and R.G. Deissler, 1961. Decay of temperature fluctuations in homogeneous turbulence before the final period. Int. J. Heat Mass Transfer, 1: 312-324.

PK, M.A.B., M.A.K. Azad and M.S.A Sarker, 2012. First-order reactant in homogeneous dusty fluid turbulence prior to the ultimate phase of decay for four-point correlation in a rotating system. Res. J. Math. Stat., 4(2): 30-38.

Sarker, M.S.A. and N. Kishore, 1991. Decay of MHD turbulence before the final period. Int. J. Eng. Sci., 29: 1479-1485. 